\title{
EPITAXIAL LAYERS VERSUS BULK SINGLE CRYSTALS OF GaN. TEMPERATURE STUDIES OF LATTICE PARAMETERS AND ENERGY GAP*
}

\author{
H. Teisseyre, P. Perlin, M. Leszczyński, T. Suski, L. Dmowski, \\ I. Grzegory, S. Porowski, J. Jun
}

High Pressure Research Center "Unipress", Polish Academy of Sciences Sokołowska 29/37, 01-142 Warszawa, Poland

AND T.D. Moustakas

Boston University, Molecular Beam Epitaxy Laboratory Department of Electrical, Computer and System Engineering Boston MA 02215, USA

\begin{abstract}
Gallium nitride epitaxial layer grown by molecular beam epitaxy and bulk crystal grown at high pressure were examined by using $\mathrm{X}$-ray diffraction methods, and by optical absorption at a wide temperature range. The free electron concentration was $6 \times 10^{17} \mathrm{~cm}^{-3}$ for the layer and about $5 \times 10^{19} \mathrm{~cm}^{-3}$ for the bulk crystal. The experiments revealed a different position of the absorption edge and its temperature dependence for these two kinds of samples. The structural examinations proved a significantly higher crystallographic quality of the bulk sample. However, the lattice constants of the samples were nearly the same. This indicated that a rather different electron concentration was responsible for the different optical properties via Burstein-Moss effect.
\end{abstract}

PACS numbers: $65.70 .+\mathrm{y}, 78.50 . \mathrm{Ge}, 78.66 . \mathrm{Fd}$

\section{Introduction}

The wide $(3.5 \mathrm{eV})$ direct energy gap of GaN makes this compound a very promising material for optoelectronics (blue lasers, detectors, light emitting diodes) and for high power and high temperature devices. The possible applications stimulate the research on the basic properties of this semiconductor. Most of the experimental works have been performed on epitaxial layers grown on foreign substrates as sapphire, gallium arsenide or silicon carbide. A high lattice mismatch between

*This work is supported by grants no. PB0288/P4/94/06 and PB066/P4/93/05 of the State Committee for Scientific Research (Republic of Poland). 
these substrates and GaN layers induces a high concentration of defects which might influence electrical and optical properties. Therefore, in our experiments we aim to compare the epitaxial layers and the bulk crystals. These crystals can be grown at high pressure (about $10 \mathrm{kbar}$ ) and high temperature (about $1300 \mathrm{~K}$ ) [1, 2]. This paper shows the results of our structural and optical examinations which led to a determination of lattice constants, thermal expansion coefficients and of temperature dependence of the energy gap for the both kinds of samples.

\section{Experiment and results}

The optical absorption measurements were performed at temperatures 10-600 K using the Spex 500M spectrometer and GaAs photomultiplier with a photon counting system. The absorption edge for the bulk crystal was shifted into higher energies by about $100 \mathrm{meV}$ as compared to the epitaxial layer. In

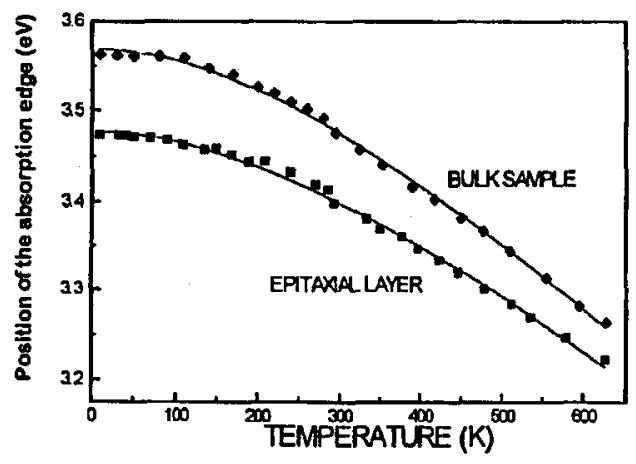

Fig. 1. Temperature variation of optical energy gap in different GaN samples.

Fig. 1 the temperature dependence of the absorption edge for the both samples is shown. The differences in optical behaviour for the bulk crystal and the layer could be attributed either to a different crystallographic perfection or to a different electron concentration which was $6 \times 10^{17} \mathrm{~cm}^{-3}$ for the layer and about $5 \times 10^{19} \mathrm{~cm}^{-3}$ for the bulk crystal. In order to check the first possibility we performed X-ray diffraction examinations by using the high resolution and the Bond diffractometers. Figure 2 shows the rocking curves of (00.4) $\mathrm{Cu} K_{\alpha_{1}}$ reflection. The full width at half maximum (FWHM) for the bulk crystals was 20-30 arcsec which indicated a very high crystallographic perfection as the theoretical FWHM for the used experimental setup was about 15 arcsec. For the GaN layer grown on sapphire substrate by molecular beam epitaxy (MBE) method the FWHM was measured as 10-14 arcmin depending on the size of the primary X-ray beam. Such a broad curve was caused by a high density of misfit dislocations which relaxed the $16 \%$ mismatch between the sapphire and gallium nitride. Figure 3 shows the temperature dependence of the GaN lattice constants (wurtzite structure) for both kinds of samples. A small difference can be observed for the lattice constant $a$ (parallel to the substrate) and it is presumably a thermal strain induced by a 


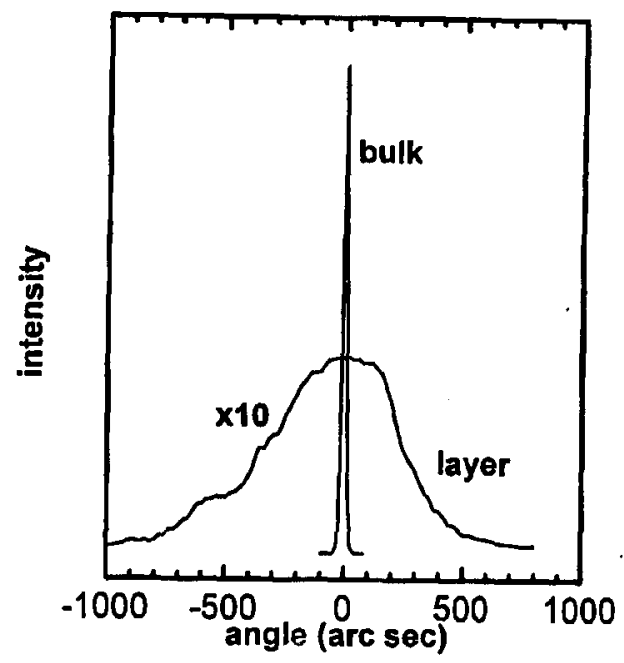

Fig. 2. X-ray rocking curve of (00.4) $\mathrm{Cu} K_{\alpha_{1}}$ reflection from $\mathrm{GaN}$ layer.
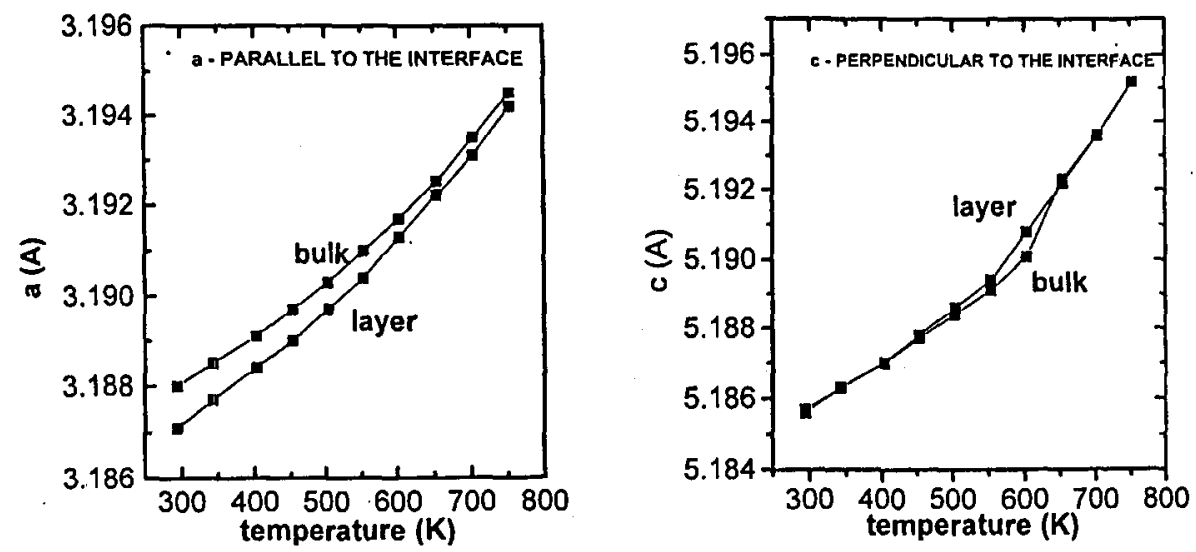

Fig. 3. Temperature dependence of GaN lattice constants (wurtzite structure).

different thermal expansion of the layer and the substrate. However, such a small strain could widen the energy gap only by about $10 \mathrm{meV}$. In our experiment we observed a much bigger effect and of the opposite sign. Therefore, we attribute the observed differences to the different free electron concentration [3]. We shall take into account that optical transitions observed in the bulk sample of $\mathrm{GaN}$ are from the top of the valence band to the unoccupied states in the conduction band near the Fermi level. For the GaN epitaxial layer the Fermi level is at the bottom of the conduction band, whereas for bulk crystal is above the conduction band minimum. In order to obtain the value of the energy gap we assumed that the absorption coefficient is expressed by $\alpha=\left(E-E_{\mathrm{g}}\right)^{1 / 2}$, where $E$ is the photon energy. This 
equation enabled us to determine the optical energy gap in our samples. The value of $E_{\mathrm{g}}$ at $20 \mathrm{~K}$ was established to be $3.43 \mathrm{eV}$. With increasing temperature the energy gap of our samples decreases mainly due to the electron-phonon interaction and the Fermi energy drops down.

\section{Summary}

We measured the absorption edge position, its temperature dependence and thermal expansion coefficient for two types of GaN crystals, bulk and epitaxial layers of different electron concentration. We found much higher crystallographic quality of the bulk crystals, which was reflected by narrower X-ray rocking curves. However, the lattice constants of the examined samples at the whole temperature range were very similar. This enabled us to draw a conclusion that the crystallographic quality is a secondary effect in the explanation of the different position of the absorption edge and its temperature variation for both kinds of gallium nitride. Instead, these differences are attributed to different electron concentration and related to Burstein-Moss effect.

\section{References}

[1] I. Grzegory, S. Krukowski, Phys. Scr. Vol. T 39, 242 (1991).

[2] M. Leszczyński, I. Grzegory, M. Bockowski, J. Cryst. Growth 126, 601 (1993).

[3] J.I. Pankove, Optical Processes in Semiconductors, Prentice-Hall, Englewood Clifts, New Jersey 1971. 